\title{
Using indentation to characterize the poroelasticity of gels
}

\section{Citation}

Hu, Yuhang, Xuanhe Zhao, Joost J. Vlassak, and Zhigang Suo. 2010. “Using Indentation to Characterize the Poroelasticity of Gels." Applied Physics Letters 96 (12): 121904. https:// doi.org/10.1063/1.3370354.

\section{Permanent link}

http://nrs.harvard.edu/urn-3:HUL.InstRepos:41461296

\section{Terms of Use}

This article was downloaded from Harvard University's DASH repository, and is made available under the terms and conditions applicable to Other Posted Material, as set forth at http:// nrs.harvard.edu/urn-3:HUL.InstRepos:dash.current.terms-of-use\#LAA

\section{Share Your Story}

The Harvard community has made this article openly available.

Please share how this access benefits you. Submit a story.

Accessibility 


\title{
Using indentation to characterize the poroelasticity of gels
}

\author{
Yuhang Hu, Xuanhe Zhao, Joost J. Vlassak, and Zhigang Suo ${ }^{\text {a) }}$ \\ School of Engineering and Applied Sciences, Harvard University, Cambridge, Massachusetts 02138, USA
}

(Received 11 December 2009; accepted 1 March 2010; published online 23 March 2010)

\begin{abstract}
When an indenter is pressed into a gel to a fixed depth, the solvent in the gel migrates, and the force on the indenter relaxes. Within the theory of poroelasticity, the force relaxation curves for indenters of several types are obtained in a simple form, enabling indentation to be used with ease as a method for determining the elastic constants and permeability of the gel. The method is demonstrated with a conical indenter on an alginate hydrogel. (C) 2010 American Institute of Physics.

[doi:10.1063/1.3370354]
\end{abstract}

Indentation has long been used to characterize diverse materials because of its ease of use and ability to probe local properties. ${ }^{1-4}$ Interest is growing to develop indentation as a method to characterize gels and tissues, soft materials ubiquitous in engineering and nature. ${ }^{5-14} \mathrm{~A}$ challenge has been to relate the response of indentation to the properties of the material.

Consider a gel that aggregates a network of crosslinked polymers and a species of mobile solvent molecules. The deformation of the gel is time-dependent, resulting from following two concurrent molecular processes: the conformational change of the network, and the migration of the solvent molecules. At a macroscopic scale, the two processes result in viscoelastic and poroelastic deformation. So long as the mesh size of the network is much smaller than the contact size of the indentation, the viscoelastic relaxation time is independent of the contact size. By contrast, the poroelastic relaxation time is quadratic in the contact size. Thus, the two types of deformation can be differentiated by indentation. Furthermore, when deformation is poroelastic, the contact can be made small to shorten the time needed for the experiment.

This paper focuses on the indentation of gels undergoing poroelastic deformation (Fig. 1). Oyen and co-workers ${ }^{11-13}$ have developed a method in which a sphere is pressed into a gel with a force ramping at a constant rate. To extract poroelastic parameters of the gel from the experimental data, the method requires a large number of precalculated master curves and a computational algorithm. By contrast, Hui et $a l^{8}$ have proposed a method in which a cylinder (in a plane-strain configuration) is pressed into a gel to a fixed depth. While this configuration is difficult to use in practice for characterizing materials, Hui and co-workers obtained a theoretical force relaxation curve in a remarkably simple form. The same form is also applicable to a cylindrical punch. $^{9}$

In this paper, we focus on spherical and conical indenters, commonly used configurations to characterize materials. We show that when these indenters are pressed into a gel to a fixed depth, the theoretical force relaxation curves also take a simple form. Consequently, the force relaxation curves can be used with ease to characterize the poroelasticity of gels. We solve for the theoretical force relaxation curves, and

${ }^{a)}$ Electronic mail: suo@seas.harvard.edu. demonstrate the method experimentally with a conical indenter on an alginate hydrogel.

To define quantities of interest, recall the theory of poroelasticity. ${ }^{15-19}$ The initial gel is taken to be in a homogenous state, subject to no mechanical load, with $C_{0}$ being the number of solvent molecules per unit volume of the gel, and $\mu_{0}$ being the chemical potential of the solvent in the gel. When the gel deforms, the displacement is a timedependent field, $u_{i}\left(x_{1}, x_{2}, x_{3}, t\right)$, and the strain is $\varepsilon_{i j}$ $=\left(\partial u_{i} / \partial x_{j}+\partial u_{j} / \partial x_{i}\right) / 2$. The conservation of solvent molecules requires that $\partial C / \partial t=-\partial J_{k} / \partial x_{k}$, where $C$ is the concentration of the solvent in the gel, and $J_{k}$ the flux.

The stress in the gel is typically so small that the volume of each molecule is commonly taken to be constant. Consequently, the increase of the volume of the gel equals the volume of the solvent absorbed, namely, $\varepsilon_{k k}=\Omega\left(C-C_{0}\right)$, where $\Omega$ is the volume per solvent molecule. When the chemical potential of the solvent in the gel changes from $\mu_{0}$ to $\mu$, the stress in the gel is given by $\sigma_{i j}=2 G\left[\varepsilon_{i j}\right.$ $\left.+\varepsilon_{k k} \delta_{i j} \nu /(1-2 \nu)\right]-\delta_{i j}\left(\mu-\mu_{0}\right) / \Omega$, where $G$ is the shear modulus, $\nu$ Poisson's ratio.

The gel is in mechanical equilibrium, so that the field of stress satisfies $\partial \sigma_{i j} / \partial x_{j}=0$. The gel, however, is not in diffusive equilibrium. The gradient of the chemical potential drives the flux of the solvent according to Darcy's law, $J_{i}=-\left(k / \eta \Omega^{2}\right) \partial \mu / \partial x_{i}$, where $k$ is the permeability and $\eta$ the viscosity of the solvent.

The volume per molecule $\Omega$ and the viscosity $\eta$ are well known for commonly used solvents. The three other parameters, $G, \nu, k$, are to be determined. A combination of the above equations leads to the familiar diffusion equation $\partial C / \partial t=D \nabla^{2} C$, with the diffusivity being $D=[2(1-\nu) /(1$ $-2 \nu)] G k / \eta$. In poroelasticity, the diffusion equation usually
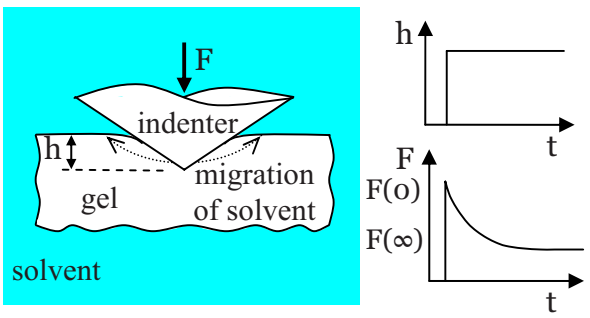

FIG. 1. (Color online) A gel is submerged in a solvent. After an indenter is pressed into the gel at a fixed depth, the solvent in the gel migrates, and the force on the indenter relaxes. 

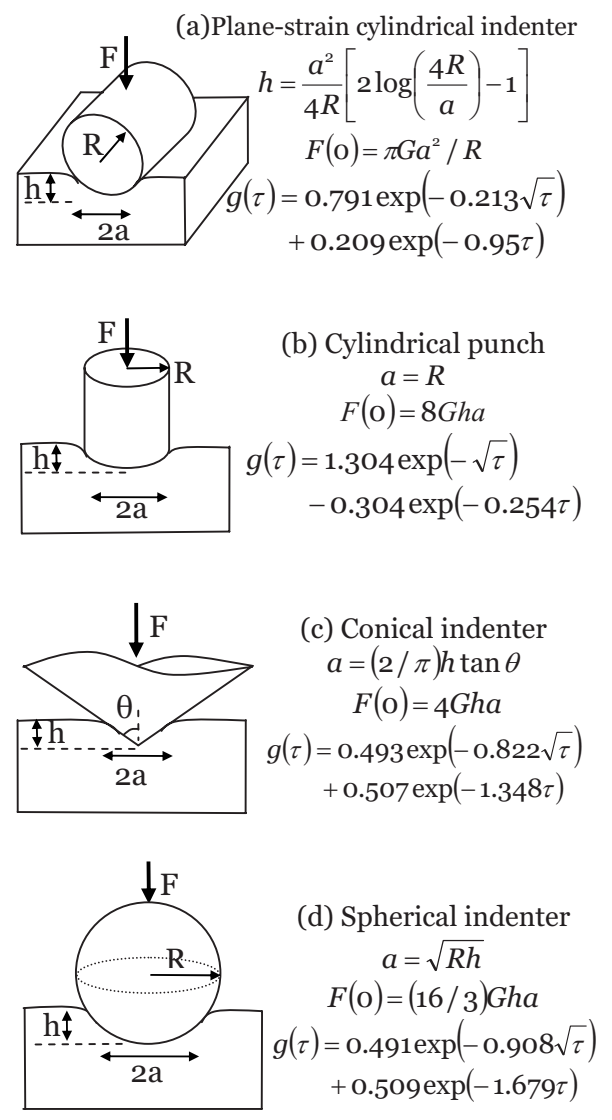

FIG. 2. Indenters of several types pressed into a gel.

cannot be solved by itself, because the boundary and initial conditions are prescribed in terms of traction, displacement, chemical potential, and flux.

As illustrated in Fig. 1, a gel is submerged in a solvent of chemical potential $\mu_{0}$. After an indenter is pressed into the gel to a fixed depth $h$, the force on the indenter relaxes as a function of time, $F(t)$. At the instance of indentation, the solvent has no time to migrate, $C=C_{0}$ and $\varepsilon_{k k}=0$, so that the gel behaves like an incompressible elastic solid, and the instantaneous force is the same as the force on an indenter pressed into an incompressible elastic solid, $F(0) \propto G$. After a long time, the solvent in the gel equilibrates with the external solvent, $\mu=\mu_{0}$, so that the gel behaves like a compressible elastic solid, and the force in equilibrium is the same as the force on an indenter pressed into a compressible elastic solid, $F(\infty) \propto G /[2(1-\nu)]$. The two limits are related as $F(0) / F(\infty)=2(1-\nu)$. Figure 2 lists the formulas of $F(0)$ for indenters of several types. ${ }^{20,21}$

For the gel to equilibrate, the solvent in the gel needs to migrate over a distance comparable to the size of the contact, $a$. At time $t$, the solvent migrates over the length scale $\sqrt{D t}$. Write the function $F(t)$ in the form

$$
\frac{F(t)-F(\infty)}{F(0)-F(\infty)}=g\left(\frac{D t}{a^{2}}\right),
$$

where $\tau=D t / a^{2}$ is the normalized time, and $g(\tau)$ is a dimensionless function specific to the type of the indenter. The ratio on the left-hand side measures how far the gel is away from the state of equilibrium. The poroelastic solution for a plane-strain cylindrical indenter exhibits two remarkable features. ${ }^{8}$ First, if the depth of the indentation, $h$, is held

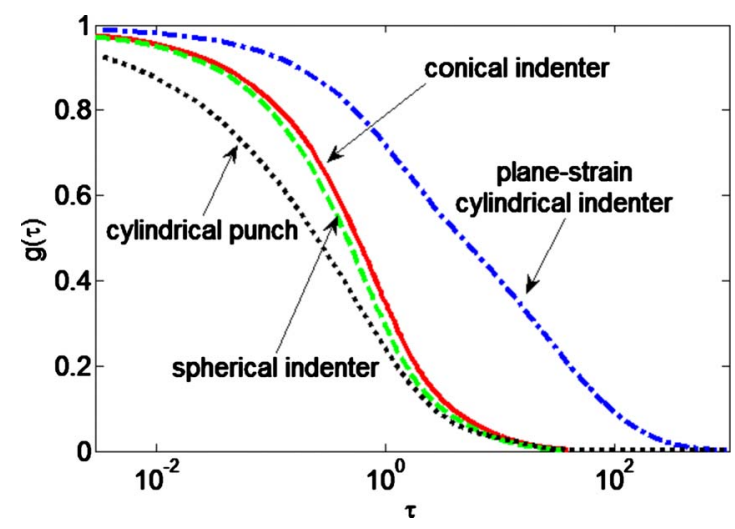

FIG. 3. (Color online) The function $g(\tau)$ for indenters of several types.

fixed while the force relaxes, the size of the contact, $a$, remains fixed over time, and the function $a(h)$ is independent of Poisson's ratio. Second, $g$ depends on all parameters in the problem through $\tau$ only. Both features are shared by the solution for a cylindrical punch. ${ }^{9}$ We next confirm that the same features are present for spherical and conical indenters.

We solve the poroelastic contact problems for indenters of several types by using the finite element software ABAQUS. In the calculation, the size of the contact is 20 times smaller than the size of the gel, and more than 16 elements are under the contact area in all calculations. Numerical results are insensitive to further increase in the size of the gel or refinement of the mesh. The initial conditions are set by the solution of an indenter pressed into an incompressible elastic solid. The boundaries of the gel outside the contact is traction-free and is in diffusive equilibrium with the external solvent, $\mu=\mu_{0}$. The indenter is taken to be rigid, frictionless, and impermeable. The calculated functions $g(\tau)$ for indenters of various types are plotted in Fig. 3, and the approximate formulas are included in Fig. 2. We have verified the two features mentioned above by varying Poisson's ratio, the half angle $\theta$ (for cones), and the ratio $R / h$ (for spheres). Our calculation reproduces the function for cylindrical punch given in Ref. 9 but does not reproduce the function for the plane-strain cylindrical indenter given in Ref. 8 .

Equation (1) and relation $F(0) / F(\infty)=2(1-\nu)$, along with the expressions for $F(0)$ and $g(\tau)$ in Fig. 2, lead to a simple method to extract the three poroelastic constants, $G, \nu, D$, from an experimentally measured relaxation curve $F(t)$. The measured value $F(0)$ determines $G$. The measured ratio $F(0) / F(\infty)$ determines $\nu$. The measured curve $F(t)$, when matched to Eq. (1), determines $D$.

We prepared a covalently crosslinked alginate hydrogel following an existing protocol. ${ }^{22}$ Each sample had a diameter of $30 \mathrm{~mm}$ and a thickness of $20 \mathrm{~mm}$, and was submerged in water during indentation. We made a conical indenter of aluminum, half angle $\theta=70^{\circ}$. The indenter was pressed into the gel to a depth of $h=1,2$, and $3 \mathrm{~mm}$. For each depth, the force was recorded as a function of time. To minimize the effect of the initial loading rate, the time used to press the indenter into the gel $(10 \mathrm{~s})$ is set to be much shorter than the relaxation time (hours).

Figure 4(a) shows that the measured relaxation curve strongly depends on the depth of indentation. For a conical indenter, the depth of indentation is the only independent length scale. ${ }^{10}$ When the force is normalized by $h^{2}$, but the 

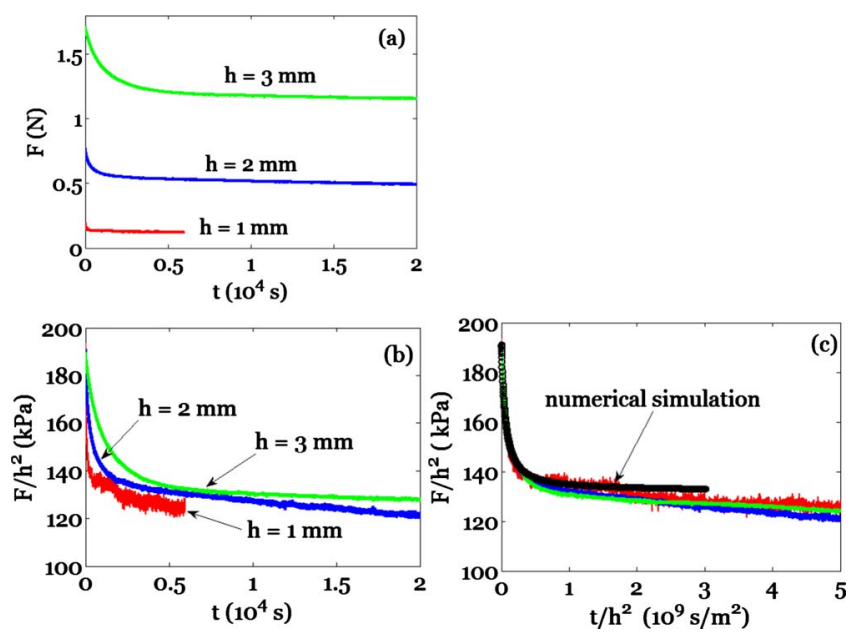

FIG. 4. (Color online) (a) Experimentally measured force as a function of time on a conical indenter pressed to three depths into a covalently crosslinked alginate hydrogel. (b) The force is normalized as $F / h^{2}$ but the time is not normalized. (c) The force is normalized as $F / h^{2}$, and the time is normalized as $t / h^{2}$.

time is not normalized, the curves for the three depths of indentation do not overlap, Fig. 4(b). Consequently, the measured relaxation curve cannot be explained by viscoelasticity. When the force is normalized as $F / h^{2}$ and the time is normalized as $t / h^{2}$, the curves for the three depths of indentation collapse into one single curve, Fig. 4(c). This behavior is consistent with poroelasticity.

A comparison of the experimental value $F(0) / h^{2}$ $=195 \mathrm{kPa}$ and the analytical formula $F(0) / h^{2}$ $=(8 / \pi) G \tan \theta$ gives the shear modulus $G=27.9 \mathrm{kPa}$. A comparison of the experimental value $F(0) / F(\infty)=1.44$ and the analytical formula $F(0) / F(\infty)=2(1-\nu)$ gives Poisson's ratio $\nu=0.28$. We used $F(\infty) / h^{2}=135 \mathrm{kPa}$ as the value for the state of equilibrium, because at this point the slope of the curve changes significantly. The slight decrease after this point is possibly caused by degradation of the network or some other mechanisms of relaxation.

The theoretical force relaxation curve $F(t)$ is given by Eq. (1), with the finite element solution of $g(\tau)$ given in Fig. 2. The theoretical curve overlaps with the experimental data in Fig. 4(c) when the diffusivity is fit to $D=3.24$ $\times 10^{-8} \mathrm{~m}^{2} / \mathrm{s}$. This value is comparable to that reported in the literature. ${ }^{23}$

The poroelastic relaxation time is quadratic in the radius of the contact, while the viscoelastic relaxation time is size- independent. Consequently, if the radius of the contact is much reduced, the effect of viscoelastic relaxation may be observed. When the contact is of millimeters, our indentation experiment shows that the relaxation behavior of the covalently crosslinked alginate hydrogel cannot be explained by viscoelasticity, but is consistent with poroelasticity. The combined poroelastic and viscoelastic relaxation at smaller scales is beyond the scope of this paper.

In summary, within the theory of poroelasticity, force relaxation curves for indenters of several types are obtained in a simple form. The experiment determines the elastic constants and permeability. The ease of use of this method will enable quantitative characterization of gels and soft tissues.

The authors acknowledge the support of the NSF (Grant No. CMMI-0800161), DARPA (Grant No. W911NF-08-10143), MRSEC at Harvard University, and Kavli Institute at Harvard University. The authors thank Li Han for the help with the indentation experiments.

${ }^{1}$ W. D. Nix, Mater. Sci. Eng., A 234, 37 (1997).

${ }^{2}$ W. C. Oliver and G. M. Pharr, J. Mater. Res. 7, 1564 (1992).

${ }^{3}$ Y. T. Cheng and C. M. Cheng, Mater. Sci. Eng., R. 44, 91 (2004).

${ }^{4}$ L. Cheng, X. Xia, L. E. Scriven, and W. W. Gerberich, Mech. Mater. 37, 213 (2005).

${ }^{5}$ D. M. Ebenstein and L. A. Pruitt, J. Biomed. Mater. Res. A 69A, 222 (2004).

${ }^{6}$ G. Constantinides, Z. I. Kalcioglu, M. McFarland, J. F. Smith, and K. J. Van Vliet, J. Biomech. 41, 3285 (2008).

${ }^{7}$ J. D. Kaufman, G. J. Miller, E. F. Morgan, and C. M. Klapperich, J. Mater. Res. 23, 1472 (2008)

${ }^{8}$ C. Y. Hui, Y. Y. Lin, F. C. Chuang, K. R. Shull, and W. C. Ling, J. Polym. Sci., Part B: Polym. Phys. 44, 359 (2006).

${ }^{9}$ Y. Y. Lin and B. W. Hu, J. Non-Cryst. Solids 352, 4034 (2006).

${ }^{10}$ W. Hong, X. Zhao, J. Zhou, and Z. Suo, J. Mech. Phys. Solids 56, 1779 (2008).

${ }^{11}$ M. L. Oyen, J. Mater. Res. 23, 1307 (2008).

${ }^{12}$ M. Galli, K. S. C. Comley, T. A. V. Shean, and M. L. Oyen, J. Mater. Res. 24, 973 (2009).

${ }^{13}$ M. Galli and M. L. Oyen, CMES 48, 241 (2009).

${ }^{14}$ S. Chiravarambath, N. K. Simha, R. Namani, and J. L. Lewis, J. Biomech. Eng. 131, 011004 (2009).

${ }^{15}$ M. A. Biot, J. Appl. Phys. 12, 155 (1941).

${ }^{16}$ J. R. Rice and M. P. Cleary, Rev. Geophys. Space Phys. 14, 227 (1976).

${ }^{17}$ G. W. Scherer, J. Non-Cryst. Solids 109, 171 (1989).

${ }^{18}$ C. Y. Hui and V. Muralidharan, J. Chem. Phys. 123, 154905 (2005).

${ }^{19}$ M. Doi, J. Phys. Soc. Jpn. 78, 052001 (2009).

${ }^{20}$ I. N. Sneddon, Int. J. Eng. Sci. 3, 47 (1965).

${ }^{21}$ K. L. Johnson, Contact Mechanics (Cambridge University Press, Cambridge, 1985).

${ }^{22}$ K. H. Bouhadir, D. S. Hausman, and D. J. Mooney, Polymer 40, 3575 (1999).

${ }^{23}$ M. Tokita and T. Tanaka, J. Chem. Phys. 95, 4613 (1991). 